\title{
The effects of familiarity on escape responses in the Trinidadian guppy (Poecilia reticulata)
}

\author{
Hayley L Wolcott ${ }^{1}$ ， Alfredo F Ojanguren ${ }^{1}$ ， Miguel Barbosa ${ }^{\text {Corresp. } 1}$ \\ ${ }^{1}$ Centre for Biological Diversity, School of Biology, University of St. Andrews, St Andrews, Fife, United Kingdom \\ Corresponding Author: Miguel Barbosa \\ Email address: mb334@st-andrews.ac.uk
}

Predation is the main cause of mortality during early life stages. The ability to avoid and evade potential threats is, therefore, favoured to evolve during the early stages of life. It is also during these early stages that the process of familiarization occurs. It has long been recognized that associating with familiar individuals confers antipredator benefits. Yet, gaps in our knowledge remain about how predator evasion is affected by social experience during early stages. In this study, we test the hypothesis that familiarization acquired during early life stages improves escape responses. Using the guppy Poecilia reticulata, we examine the effect of different recent social conditions in the three main components of predator evasion. Using high-speed motion analysis, we compared the number of individuals in each test group that responded to a visual stimulus, their reactive distance and magnitude of their response (maximum speed, maximum acceleration and distance) in groups composed either of familiar or non-familiar individuals. Contrary to the prediction, groups composed of familiar individuals were less responsive than groups of unfamiliar individuals. Reactive distance and magnitude of response were more dependent on individual size rather than on familiarity. Larger individuals reached higher maximum speeds and total distances in their escape response. Our result indicates that familiarity is likely to affect behaviour earlier in a predator-prey interaction, which then affects the behavioural component of the response. Taken together our study contributes to previous ones, by distinguishing which components of an escape response are modulated by familiarity. 
1 The effects of familiarity on escape responses in the Trinidadian guppy

2 (Poecilia reticulata)

3

4 Hayley L Wolcott ${ }^{1}$, Alfredo F Ojanguren ${ }^{1} \&$ Miguel Barbosa $^{1}$

5

$6{ }^{1}$ Centre for Biological Diversity, School of Biology,

7 University of St. Andrews,

8 St Andrews,

9 Fife,

10 KY16 9TF

11 UK

12

13 Corresponding Author:

14 Miguel Barbosa ${ }^{1}$

15 Email address: mb334@st-andrews.ac.uk 


\section{ABSTRACT}

18 Predation is the main cause of mortality during early life stages. The ability to avoid and evade 19 potential threats is, therefore, favoured to evolve during the early stages of life. It is also during 20 these early stages that the process of familiarization occurs. It has long been recognized that 21 associating with familiar individuals confers antipredator benefits. Yet, gaps in our knowledge

22 remain about how predator evasion is affected by social experience during early stages. In

23 this study, we test the hypothesis that familiarization acquired during early life stages improves 24 escape responses. Using the guppy Poecilia reticulata, we examine the effect of different recent 25 social conditions in the three main components of predator evasion. Using high-speed motion 26 analysis, we compared the number of individuals in each test group that responded to a visual 27 stimulus, their reactive distance and magnitude of their response (maximum speed, maximum 28 acceleration and distance) in groups composed either of familiar or non-familiar individuals. 29 Contrary to the prediction, groups composed of familiar individuals were less responsive than 30 groups of unfamiliar individuals. Reactive distance and magnitude of response were more 31 dependent on individual size rather than on familiarity. Larger individuals reached higher 32 maximum speeds and total distances in their escape response. Our result indicates that familiarity 33 is likely to affect behaviour earlier in a predator-prey interaction, which then affects the 34 behavioural component of the response. Taken together our study contributes to previous ones, 35 by distinguishing which components of an escape response are modulated by familiarity. 

organisms are at heightened risk due to their smaller size (Cushing 1974). Natural selection is

41

42 43 therefore expected to favour the development of antipredator behaviours early in life

(Braithwaite \& Salvanes 2005; Vilhunen \& Hirvonen 2003). Antipredator behaviours are generally divided into two major types; 1) avoidance and 2) evasion (Fuiman \& Magurran 1994; reduces the likelihood of encountering a predator and consequently of its attack (Fuiman \& Magurran 1994). Evasion, on the other hand, occurs once the predator initiates the attack. As predator avoidance is not always possible, successful predator evasion tactics are essential for survival. The behaviour and frequency at which each evasion tactic is employed is contextdependent; individuals adopt behaviours that improve their evasive response and, thus, enhance survival (Domenici 2010).

One way in which organisms may reduce the risk of predation is by associating with others, either by schooling or just by joining a group (Ruxton \& Johnsen 2016; Ward \& Webster 2016). Though groups might be more conspicuous to a predator, each individual within the group has a smaller probability of being predated than if alone. Among the group antipredator benefits of enhanced vigilance, dilution of risk, predator confusion and coordinated antipredator maneuverers (Krause \& Ruxton 2002; Ward \& Webster 2016), there is strong evidence showing that familiarity within the group enhances antipredator behaviours (Griffiths et al. 2004). Familiarity between conspecifics can be broadly defined as the ability to discriminate between individuals based on previous interactions (Griffiths 2003). The process of familiarization is based on visual, and auditory and olfactory cues (Coffin et al. 2011; Reby et al. 2001; Zajitschek \& Brooks 2008). Repeated exposure to a stimulus can lead to familiarisation, in a social context 
62 that may be conspecifics with whom an individual interacts, such as during foraging. Fitness

63 benefits of joining a group composed of familiar conspecifics over unfamiliar individuals has

64 been demonstrated in various taxa (Figueroa et al. 2013; Grabowska-Zhang et al. 2012;

65 Grabowska-Zhang et al. 2011; Strodl \& Schausberger 2012; Strodl \& Schausberger 2013),

66 particularly in shoaling fish (Barber \& Wright 2001; Griffiths \& Magurran 1997b)

The benefits in associating with familiar individuals for the social learning and for the development and acquisition of successful antipredator responses in shoaling fish are acknowledged (Swaney et al. 2001; Ward \& Hart 2003). Groups composed by familiar individuals may be more cohesive and have reduced neighbour distance (Chivers et al. 1995; Höjesjö et al. 1998), characteristics which enhance predator confusion and dilute individual risk. Further, familiar groups generally experience reduced within-group aggression and evolve more stable social hierarchies (Griffiths et al. 2004; Höjesjö et al. 1998; Johnsson 1997; Tanner \&

74 Keller 2012). Reduced aggression within familiar groups allows more time for predator 75 vigilance, which may improve escape latency (Griffiths et al. 2004; Strodl \& Schausberger 2012). Additionally, individuals are more likely to perform cooperative antipredator behaviours when in familiar groups, as they may remember whether the others have behaved cooperatively in the past (Dugatkin \& Alfieri 1991). For example, individuals in familiar groups may be more likely to perform more risky antipredator manoeuvres (Chivers et al. 1995), join predator mobbing (Grabowska-Zhang et al. 2012), or perform predator inspection (Dugatkin \& Godin 1992). Such antipredator behaviours put individuals at higher risk, but improve group 82 antipredator response. While the effect and importance of familiarity on predator avoidance is well recognised, 84 how familiarity shapes predator evasion, particularly the escape response, remains largely 
85 unexplored For example, studies to date have focused exclusively on the effect of familiarity on

86 the latency of the response (Griffiths et al. 2004; Strodl \& Schausberger 2012) and have not

87 considered other aspects of the escape performance. Successful escape responses depend on

88 various components, such as latency, velocity and distance travelled in the response (Domenici

$89 \&$ Blake 1997). For instance, latency, considered as the time between the onset of the predator

90 attack and the start if the response, is crucial for the outcome of the interaction (Fuiman et al.

91 2006). Also, an effective response requires moving away from the attack trajectory fast enough

92 so the predator cannot adjust it (Fuiman \& Cowan 2003). Studies on escape behavior have

93 focused on the aspects of the escape response which are modulated by the relative cost of

94 escaping and perceived risk, such as latency, reactive distance (the distance between the predator

95 and prey when the prey initiates a response) and responsiveness (whether or not a prey responds

96 to an attack) (Domenici 2010). Kinematic aspects of escape responses, on the other hand, are less

97 often considered, as they have been considered to be constrained by the sensory-motor system of

98 the individual (Domenici \& Blake 1997). A review by Domenici (2010) emphasizes that

99 performance in escape responses is not always maximized to the physical capabilities of the

100 individual, which suggests that other factors may cause variability in escape responses. Given the

101 importance of social behaviour in reactive distance and responsiveness (Dial et al. 2016), it is

102 plausible that the kinematic aspects of an escape response may be modulated in a similar way by

103 familiarity. In order to fully assess the escape performance of fish, we need to employ an

104 approach that takes into account the multiple behavioural aspects on an escape response. The aim

105 of this study was to address the role of familiarity acquired during early life stages in affecting

106 the different components of the antipredator escape responses in the Trinidadian guppy (Poecilia 107 reticulata). 

important for the establishment and reinforcement of individual discrimination and familiarity in

110 guppies (Barbosa et al. 2016; Barbosa et al. 2013; Chapman et al. 2008a; Chapman et al. 2008b;

111 Laland et al. 2003). Within group familiarity is likely to affect how a group of individuals

112 respond to a potential predator. Guppies respond to a predator attack by performing a "fast-start"

113 escape response, characteristic to most fish species (Dial et al. 2016). This evasion tactic consists

114 of an unambiguous quick and sudden burst of swimming activity usually of only tenths of a

115 second that propels the fish away from an oncoming predator (Domenici \& Blake 1997; Fuiman

116 et al. 2010; Webb 1978; Weihs 1973). Fast-start escape responses integrate a combination of

117 behavioural and kinematic components (Marras et al. 2011), both of which were examined in this 118 study.

In view of the antipredator benefits of familiarity, we predicted that juvenile guppies are

120 also more responsive and perform more successful escape responses when in groups of familiar

121 conspecifics. To test this prediction, we exposed familiar and unfamiliar groups of juvenile

122 guppies to a digital display of a looming object and quantified the difference in responsiveness

123 (number of fish responding), reactive distance (based on the size of the stimulus when the

124 response started) and magnitude of the escape response (maximum speed and acceleration

125 achieved during the response, and distance covered by the escaping fish). This approach allows

126 us to identify the role of familiarity in a behaviour closely related to survival during early life

127 stages and to pinpoint which components of an escape response are more likely to be affected by 128 social experience. 


\section{METHODS}

All guppies used were $8^{\text {th }}$ generation descendants of individuals collected from the Lower

132 sections of the Tacarigua River in Trinidad. Several species of fish predators have been reported

133 in this locality including the pike cichlid (Crenicichla alta), the blue acara (Aequidens pulcher)

134 and the wolf fish (Hoplias malabaricus), which also prey intensively on juvenile guppies

135 (Magurran \& Seghers 1994). Experimental fish were housed, and all observations recorded, at

136 the aquarium facility at the Sir Harold Mitchell Building, University of St Andrews, UK. The

137 aquarium has an air temperature control system, which kept the tank temperatures at a mean

$138( \pm \mathrm{SD})$ temperature of $24.5^{\circ} \mathrm{C}\left( \pm 0.3^{\circ} \mathrm{C}\right)$. All stock tanks contained similar numbers of males,

139 females and juveniles. Lighting conditions followed a 12-hour light/dark cycle. All fish were fed

140 daily with TetraMin ${ }^{\circledR}$ flake food. Our experimental design was examined by the Biology School

141 Ethics Committee from the University of St Andrews and declared our study to be exempted of

142 Animal Ethics approval.

143

$144 \quad$ Test Fish Collection and Rearing

145 Prior to the experiment, we collected three juveniles from three different stock tanks (60

$146 \times 40 \times 40 \mathrm{~cm}$ ) that contained a mix of males, females and juveniles using a dip net (there are 15

147 Lower Tacarigua stock tanks in at the University of St Andrews aquarium facility). This ensured

148 that the test groups were composed neither of familiar conspecifics nor of close kin. Further, in

149 all stock tanks there are large and smaller boulders and java moss, which allows a more natural

150 environment for guppies. Immature juvenile guppies (i.e. age between 5 and 6 weeks) were

151 allocated to a holding tank $(20 \times 22 \times 30 \mathrm{~cm})$ a to create a test group. Each test group was 
152 composed of three individuals. A total of 42 holding tanks were used. Black plastic sheets were

153 placed between each tank to ensure each test group was visually isolated from adjacent groups.

154 Fish were of similar size and randomly distributed between holding tanks (mean $( \pm \mathrm{SD}) 10.8( \pm$

155 1.7) $\mathrm{mm}$ ). Nevertheless, in order to be able to identify each individual during tracking, test

156 groups were carefully constituted of different sized individuals. Each test group remained in its

157 holding tank for two weeks to ensure the establishment of familiarity between tank mates

158 (Griffiths \& Magurran 1997a).

\section{Escape Response Trials}

160

We split the juveniles into two treatments: a familiar and an unfamiliar. Each group was

161 composed of three juveniles (Total 42 groups, 21 familiar and 21 unfamiliar). Each day we tested

162 six groups, three groups with familiar individuals and three of unfamiliar individuals. In familiar

163 groups, individuals were tested with those fish that they shared the holding tank with for two

164 weeks prior to testing. For unfamiliar groups, we took three fish, each from a different holding

165 tank so they had not seen each other before, and put them together in the observation chamber

166 for testing (Figure 1). Unfamiliar groups were treated as a control. Each group was only tested 167 once.

All tests occurred between 9:00 and 11:00 am and at least an hour after being fed. These

169 measures were taken to avoid differences in satiation rate and time of day that might affect the

170 behaviour of the individuals. The experimental setup used to assess escape response was based

171 on an established protocol (Fuiman et al. 2010), but modified for this experiment (Figure 2).

172 Each trial involved presenting a digital display of a looming object to a test group. The digital

173 display consists 1.8-second sequence showing black oval in the middle of a white background

174 that increases its size to simulate an approaching object (Supplementary Information). The same 
175 stimulus has been shown to elicit a startle response in larval fish of similar size (Fuiman et al.

176 2006; Ojanguren \& Fuiman 2010). The video was presented using a LCD screen (Braun 1210)

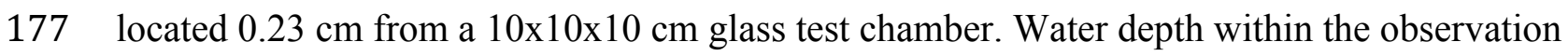

178 chamber was kept at $225 \mathrm{ml}$ to minimise vertical movement in escape responses. For each trial, a

179 test group was transported to the observation chamber one individual at a time and given at least

18010 minutes of acclimatisation to their new surroundings before testing began. Each individual

181 fish was only tested once. After the terminus of the trials the individuals were returned to a stock

182 tank and were not reused in the experiment.

183 Individual response to the visual stimulus was recorded at 240 frames $\mathrm{s}^{-1}$ using a high-

184 speed video camera (Casio EX-FH25 EXILM) through a 45'-angled mirror to obtain an overhead

185 view of the observation chamber. The observation chamber sat on top of a black surface and was

186 illuminated by lamps positioned left and right of the chamber so that the response could be

187 clearly observed. All individuals tested were transferred to a small petri dish with a small amount

188 of water and photographed from above. Individual standard length was measured to nearest

189 millimetre using ImageJ analysis software (Abràmofff et al. 2004). All tested individuals

190 resumed normal routine swimming activity immediately after the escape responses. No fish died

191 during the tests, or after the picture was taken. After the terminus of the study, all individuals

192 were returned to stock tanks.

193

194 Data Analysis

195 Video recordings were analysed frame by frame to determine responsiveness (the number

196 of fish that responded to the stimulus in each test group) and the reactive distance (the virtual

197 distance between the looming object and the first individual that responded, calculated from the 
198 size of the oval on the screen at the moment of the start of the response and the distance of the

199 fish from the screen) (see (Fuiman et al. 2010) for details). The position of the fish in 2-

200 dimensional coordinates for the overhead view was obtained using the manual tracking plugin in

201 ImageJ (Cordelières 2005), this allowed us to calculate maximum speed, maximum acceleration

202 and total distance covered in the response (see (Fuiman et al. 2010; Fuiman et al. 2006)).

203

204 Statistical Analysis

205

Differences in responsiveness between familiar and unfamiliar groups were tested with a

206 Wilcoxon rank sum test to account for the fact that responsiveness was a discrete variable. The

207 responsiveness of each test group was ranked according to the number of individuals within the 208 group that responded (either $0,1,2$ or 3 ). We considered that the response was over when the 209 distance travelled between three consecutive frames (12.5 milliseconds) was $1 \mathrm{~mm}$ or less.

210 In order to investigate the effect of familiarity on reactive distance and in the magnitude

211 of the response (maximum speed, maximum acceleration and distance covered in a response) we

212 used Generalised Linear Fixed Effect Models (GLM). Reactive distance, maximum speed,

213 maximum acceleration and distance travelled during a response were only measured on the first

214 fish that responded. On the only trial that two fish responded in the same frame, the fish that had

215 the larger reactive distance was considered the first responder. Each group was only tested once.

216 Each full model included familiarity as main effect treatment and standard length as a covariate

217 (i.e. of the individual that first responded), as well as their interaction. The linear predictor and

218 expected values scales were linked using a log function. Diagnostic plots revealed significant

219 departures from normality of the residuals for both response variables reactive distance and total 220 distance. Normality and homogeneity assumptions about the distributions of residual values on 
221 the dependent variable were improved by log-transforming the response variables. To account

222 for the effect of size in escape responses, all models included individual standard length as

223 covariate. All analyses were performed in using R (Team 2016).

224

225 RESULTS

226 Individual standard length between familiar and unfamiliar treatments did not differ (mean $( \pm$

227 SD), Familiar $=117.3(19.7) ;$ Unfamiliar $=122.2(27.9), p=0.089)$

\section{Responsiveness}

229 A total of 42 groups composed of three different sized individuals were tested. Of the 30 230 groups in which one or more individuals responded, 19 groups were familiar and 16 groups were 231 unfamiliar. There was a significant effect of familiarity on responsiveness (Wilcoxon rank sum: $232 \mathrm{~W}=451.5, p<0.001$ ) (Figure 3), where responsiveness was higher in unfamiliar groups. In the 233 majority of familiar groups only one individual in the group responded, whereas the unfamiliar 234 groups showed more instances where two or more individuals reacted to the stimulus.

\section{Reactive distance}

237 We failed to detect an effect of familiarity and individual standard length on reactive distance 238 (Table 1, Figure 4A). 


\section{Magnitude of the response}

241 We failed to detect an effect of social treatment on maximum speed $(p=0.263)$,

242 maximum acceleration $(p=0.699)$ and total distance $(p=0.698)$ (Table 1, Figure 4$)$. For

243 maximum acceleration the effect of individual standard length was similar between treatments $(p$

$244=0.078)$. There was an increased in both maximum speed and total distance travelled as a

245 function on increased standard length (Figure 4C, D, Table 1). Notably, we observed an almost

246 twice-greater gradient in the familiar treatment than in the unfamiliar treatment in terms of

247 maximum speed. There was an increase of 82.05 milliseconds in maximum speed per millimetre

248 of standard size in the familiar treatment, while in the unfamiliar treatment the gradient was of

24944.14 milliseconds per millimetre of standard size (Table 1, Figure 4B).

250

251 DISCUSSION

252 A novel contribution of this study is that it examines the consequences of familiarity

253 during early stages in the performance of escape responses separating the multiple aspects of the

254 response to determine which parts depend on the social environment. Through high-speed

255 analysis of the escape responses in familiar and unfamiliar groups of guppies, we demonstrate

256 that early social experience plays a role in shaping how groups of fish respond to a stimulus.

257 Namely, we showed that unfamiliar groups had more individuals perform an escape response

258 than those in familiar groups. Unexpectedly, other components of the escape response, namely

259 latency and magnitude, were not affected by familiarity. Furthermore, the maximum speed and

260 distance covered in the response were correlated with individual size rather than with level of 
261 familiarity within the group. In combination, our study suggests, that familiarity plays a less

262 meaningful role in determining some behavioural components of the escape response. number of individuals responding within each group among unfamiliar groups than among avoid a potential predator (Domenici 2010; Fuiman \& Magurran 1994), escape responses may however vary within and among individuals (Lima \& Dill 1990; Ward \& Webster 2016;

268 Ydenberg \& Dill 1986). If there is enough information to accurately predict the level of threat in 269 a given environment, then it is advantageous for a prey to only flee when it is necessary for 270 survival avoiding false alarms that could in turn attract the attention of nearby predators (Ward et 271 al. 2011). For example, minnows performed antipredator behaviours in response to a realistic 272 pike model, whereas an unrealistic stimulus elicited no response (Magurran \& Girling 1986).

273 The lower responsiveness in familiar groups may be a result of improved vigilance. According to 274 the theory of limited attention, performance is reduced when attention must be divided among 275 different tasks (Dukas 2002). Therefore, if individuals are not spending time inspecting or acting 276 aggressively toward group mates, as is often found among unfamiliar individuals (Griffiths et al. 277 2004; Johnsson 1997; Tanner \& Keller 2012), then they are likely to have more time to dedicate 278 to other tasks, such as predator vigilance (Strodl \& Schausberger 2012; Strodl \& Schausberger 279 2013; Zach et al. 2012). Guppies from familiar groups may have been able to accurately assess 280 the non-threatening nature of the stimulus. An alternative explanation is that fish in familiar 281 groups feel safer as they are with individuals they have seen before and this may be why familiar 282 individuals are more likely to perceive the oval shape stimulus as non-threatening. On he other 283 hand unfamiliar groups may have been more skittish and, thus more likely to be startled by the 
284 stimulus. Interacting with unfamiliar individuals can be stressful (Choleris et al. 1998),

285 particularly if such interactions are associated with increased aggression (Galef et al. 1984).

286 Individuals may perceive higher risk when shoaling with unfamiliar conspecifics, as was found

287 in fathead minnows who had a higher production of epidermal alarm substance cells when in 288 unfamiliar shoals than familiar shoals (Wisenden \& Smith 1998). Furthermore, escape responses

289 from the digital display may be misinterpreted as an attack by the other group mates. Aggression 290 is common among guppies, in both natural as well as laboratory conditions (Magurran 2005;

291 Thibault 1974). Therefore, it is plausible that an individual guppy would flee from an unfamiliar 292 group mate that is performing a fast-start response, as this could be misinterpreted as an attack.

We failed to detect an effect of familiarity on the reactive distance of an escape response.

294 Comparable studies have found that familiarity reduces the latency of an escape response.

295 Similarly, familiar juvenile brown trout responded $14 \%$ faster than unfamiliar ones when 296 exposed to a simulated predator attack (Griffiths et al. 2004). In both their and our study 297 reduction in reaction time has been attributed to the associated benefits of the theory of limited 298 attention. Our results, therefore, suggest that familiarity is more important in antipredator 299 behaviours earlier in a predator sequence. A predator must successfully encounter, attack and 300 capture a prey, where a prey's strategy is to interrupt this sequence. It has been suggested that 301 avoiding the encounter and attack, are prey's best strategy (Fuiman \& Magurran 1994). Previous 302 experiments included an entire predator interaction, such as a model heron swinging forward and 303 plunging its beak into the water (Griffiths et al. 2004) or a live predator (Strodl \& Schausberger 304 2012), and could, therefore elicit such behaviours. In contrast, our experiment only elicited 305 behaviours seen in the last few milliseconds of the attack. 
Familiarity has been found to enhance avoidance tactics. For example, predator confusion was enhanced in shoals of familiar fathead minnows that had reduced neighbour distance and more shoal cohesion in response to predator stimuli compared to unfamiliar shoals (Chivers et al. 1995). Tighter shoal cohesion reduces the probability of being captured by a predator (Mathis \& Smith 1993). In addition, familiar shoals exhibited a greater number of predator inspections with more inspectors per inspection when faced with a model pike (Chivers et al. 1995). Predator inspection, where an individual or small group of individuals approach a predator, pause and swim away (Pitcher 1991), enables prey to gain valuable information on the threat of a predator.

314 This behaviour, though risky to inspectors, is associated with improved avoidance of a predator attack (Godin \& Davis 1995; Magurran 1990; Magurran \& Pitcher 1987). Therefore, it is likely that familiarity is more crucial in antipredator behaviour associated with predator avoidance than predator evasion.

The effect of familiarity on the magnitude of the response was not significant. The kinematic aspects of escape responses are often assumed to be constrained by the sensorymotor system of the individual (Domenici \& Blake 1997). However, juvenile guppies reared in an environment with intense social aggression travelled a greater distance in the

322 first five frames after a simulated avian attack than those reared in absence of social 323 aggression (Chapman et al. 2008a). It is then recognized the need to implement an integrative

324 approach that accounts for all aspects of an escape response in order to obtain a clear 325 understanding of the mechanisms of response to a predator (Domenici 2010). While other 326 behavioural variables may affect the magnitude of an escape response, our study provides 327 evidence that familiarity is not one of them. Our results showed that size, rather than familiarity, 328 influenced the magnitude of the response than familiarity. This result is consistent with previous 
329 studies that have shown that the magnitude of the fast-start response in young fish increases with

330 body length (Dial et al. 2016). While behavioural effects on the locomotive performance cannot

331 be ruled out (Domenici 2010), our study and others (Gibb et al. 2006; Ojanguren \& Braña 2003)

332 lend strong support that the magnitude of a fast-start response is largely determined by

333 morphology, rather than by social conditions.

334 In this study, we provided a test for the relative effect of familiarity in modulating

335 predator avoidance behaviour by measuring several components of the escape responses using

336 high speed video analysis. Our results suggest that familiar groups may have improved

337 antipredator performance, as individuals conserve energy and are less conspicuous by not fleeing

338 in a non-threatening situation. Nevertheless, further studies are necessary to elucidate this. Future

339 studies may try to tease the contribution of group size and familiarity in modulating the predator

340 escape response, by testing familiar and non-familiar groups composed of different number of

341 individuals. Our results also suggest that the effects of familiarity on the response are perhaps

342 unlikely to play a role on escape performance in the last few milliseconds of a predator attack.

343 Instead, we believe that familiarity is more likely to affect behaviour earlier in a predator-prey

344 interaction, which then affects the quality of the response.

\section{ACKNOWLEDGMENTS}

347 We are grateful to Maria Dornelas, Anne Magurran and Mike Webster for providing

348 helpful comments on early drafts. We are also thankful to Maria Joao Janeiro for helping with

349 the statistics and to the Biodiversity and Behaviour Group for the rewarding discussions and 350 comments throughout this research. 
353

354

355

356

357

358

359

360

361

362

363

364

365

366

367

368

369

370

371

372

373

374

375

376

377

378

379

380

381

382

383

384

385

386

387

388

389

390

391

392
Abràmofff MD, Magalhães PJ, and Ram SJ. 2004. Image processing with ImageJ. Biophotonics International 11:36-43.

Barber I, and Wright HA. 2001. How strong are familiarity preferences in shoaling fish? Animal Behaviour 61:975-979. http://dx.doi.org/10.1006/anbe.2000.1665

Barbosa M, Camacho-Cervantes M, and Ojanguren AF. 2016. Phenotype matching and early social conditions affect shoaling and exploration decisions Ethology 122:171-179. $10.1111 /$ eth. 12455

Barbosa M, Ojanguren AF, and Magurran AE. 2013. Courtship display persists despite early social deprivation. Ethology 16:496-502.10.1111/eth.12087

Braithwaite VA, and Salvanes AGV. 2005. Environmental variability in the early rearing environment generates behaviourally flexible cod: implications for rehabilitating wild populations. Proceedings of the Royal Society B-Biological Sciences 272:11071113. 10.1098/rspb.2005.3062

Chapman BB, Morrell LJ, Benton TG, and Krause J. 2008a. Early interactions with adults mediate the development of predator defenses in guppies. Behavioral Ecology 19:87-93.10.1093/beheco/arm111

Chapman BB, Ward AJW, and Krause J. 2008b. Schooling and learning: early social environment predicts social learning ability in the guppy, Poecilia reticulata. Animal Behaviour 76:923-929. 10.1016/j.anbehav.2008.03.022

Chivers DP, Brown GE, and Smith RJF. 1995. Familiarity and shoal cohesion in fathead minnows (Pimephales promelas): implications for antipredator behaviour. Canadian Journal of Zoology 73:955-960. 10.1139/z95-111

Choleris E, Valsecchi P, Wang Y, Ferrari P, Kavaliers M, and Mainardi M. 1998. Social Learning of a Food Preference in Male and Female Mongolian Gerbils is Facilitated by the Anxiolytic, Chlordiazepoxide. Pharmacology Biochemistry and Behavior 60:575-584. http://dx.doi.org/10.1016/S0091-3057(98)00005-7

Coffin HR, Watters JV, and Mateo JM. 2011. Odor-Based Recognition of Familiar and Related Conspecifics: A First Test Conducted on Captive Humboldt Penguins (<italic $>$ Spheniscus humboldti</italic $>$ ). PLoS One 6:e25002. 10.1371/journal.pone.0025002

Cordelières FP. 2005. Manual Tracking. http://rsbweb.nih.gov/ij/plugins/track/Manual

Cushing DH. 1974. The possible density-dependence of larval mortality and adult mortality in fishes. In: Blaxter J, editor. The Early Life History of Fish. New York: SpringerVerlag.

Dial TR, Reznick DN, and Brainerd EL. 2016. Effects of neonatal size on maturity and escape performance in the Trinidadian guppy. Functional Ecology 30:943-952. 10.1111/1365-2435.12565

Domenici P. 2010. Context-dependent variability in the components of fish escape response: integrating locomotor performance and behavior. Journal of Experimental Zoology Part A: Ecological Genetics and Physiology 313A:59-79. 10.1002/jez.580 
393 Domenici P, and Blake R. 1997. The kinematics and performance of fish fast-start

394

395

396

397

398

399

400

401

402

403

404

405

406

407

408

409

410

411

412

413

414

415

416

417

418

419

420

421

422

423

424

425

426

427

428

429

430

431

432

433

434

435

436

437 swimming. The Journal of Experimental Biology 200:1165-1178.

Dugatkin LA, and Alfieri M. 1991. Guppies and the TIT FOR TAT strategy: preference based on past interaction. Behavioral Ecology and Sociobiology 28:243-246. $10.1007 / \mathrm{bf00175096}$

Dugatkin LA, and Godin J-GJ. 1992. Prey approaching predators: a cost-benefit perspective. Annales Zoologici Fennici 29:233-252.

Dukas R. 2002. Behavioural and ecological consequences of limited attention. Philosophical Transactions of the Royal Society B: Biological Sciences 357:1539-1547. 10.1098/rstb.2002.1063

Figueroa J, Solà-Oriol D, Manteca X, and Pérez JF. 2013. Social learning of feeding behaviour in pigs: Effects of neophobia and familiarity with the demonstrator conspecific. Applied Animal Behaviour Science 148:120-127. 10.1016/j.applanim.2013.06.002

Fuiman LA, and Cowan JH. 2003. Behavior and recruitment success in fish larvae: repeatability and covariation of survival skills. Ecology 84:53-67. 10.1890/00129658(2003)084[0053:BARSIF]2.0.CO;2

Fuiman LA, and Magurran AE. 1994. Development of predator defences in fishes. Reviews in Fish Biology and Fisheries 4:145-183. 10.1007/bf00044127

Fuiman LA, Meekan MG, and McCormick MI. 2010. Maladaptive behavior reinforces a recruitment bottleneck in newly settled fishes. Oecologia 164:99-108. 10.1007/s00442-010-1712-3

Fuiman LA, Rose KA, Cowan JH, and Smith EP. 2006. Survival skills required for predator evasion by fish larvae and their relation to laboratory measures of performance. Animal Behaviour 71:1389-1399. http://dx.doi.org/10.1016/j.anbehav.2005.11.013

Galef BG, Kennett DJ, and Wigmore SW. 1984. Transfer of information concerning distant foods in rats: A robust phenomenon. Animal Learning \& Behavior 12:292-296. $10.3758 /$ bf03199970

Gibb Alice C, Swanson Brook O, Wesp H, Landels C, and Liu C. 2006. Development of the Escape Response in Teleost Fishes: Do Ontogenetic Changes Enable Improved Performance? Physiological and Biochemical Zoology 79:7-19. 10.1086/498192

Godin J-GJ, and Davis SA. 1995. Who Dares, Benefits: Predator Approach Behaviour in the Guppy (Poecilia reticulata) Deters Predator Pursuit. Proceedings of the Royal Society of London B: Biological Sciences 259:193-200. 10.1098/rspb.1995.0028

Grabowska-Zhang AM, Sheldon BC, and Hinde CA. 2012. Long-term familiarity promotes joining in neighbor nest defence. Biology Letters. 10.1098/rsbl.2012.0183

Grabowska-Zhang AM, Wilkin TA, and Sheldon BC. 2011. Effects of neighbor familiarity on reproductive success in the great tit (Parus major). Behavioral Ecology. $10.1093 /$ beheco/arr189

Griffiths SW. 2003. Learned recognition of conspecifics by fishes. Fish and Fisheries 4:256268. 10.1046/j.1467-2979.2003.00129.x

Griffiths SW, Brockmark S, Höjesjö J, and Johnsson JI. 2004. Coping with divided attention: the advantage of familiarity. Proceedings of the Royal Society of London B: Biological Sciences 271:695-699. 10.1098/rspb.2003.2648

Griffiths SW, and Magurran AE. 1997a. Familiarity in schooling fish: how long does it take to acquire? Animal Behaviour 53:945-949. 10.1006/anbe.1996.0315 
438 Griffiths SW, and Magurran AE. 1997b. Schooling Preferences for Familiar Fish Vary with

439

440

441

442

443

444

445

446

447

448

449

450

451

452

453

454

455

456

457

458

459

460

461

462

463

464

465

466

467

468

469

470

471

472

473

474

475

476

477

478

479

480

481

482
Group Size in a Wild Guppy Population. Proceedings of the Royal Society of London B: Biological Sciences 264:547-551.

Höjesjö J, Johnsson JI, Petersson E, and Järvi T. 1998. The importance of being familiar: individual recognition and social behavior in sea trout (Salmo trutta). Behavioral Ecology 9:445-451. 10.1093/beheco/9.5.445

Johnsson JI. 1997. Individual Recognition Affects Aggression and Dominance Relations in Rainbow Trout, Oncorhynchus mykiss. Ethology 103:267-282. 10.1111/j.14390310.1997.tb00017.x

Krause J, and Ruxton GD. 2002. Living in groups. In: Evolution OSiEa, editor: New York: Oxford University Press. p 228.

Laland KN, Brown C, and Krause J. 2003. Learning in fishes: from three-second memory to culture. Fish and Fisheries 4:199-202. 10.1046/j.1467-2979.2003.00124.x

Lima SL, and Dill LM. 1990. Behavioural decisions made under the risk of predation: a review and prospectus. Canadian Journal of Zoology 68:619-640.

Magurran AE. 1990. The adaptive significance of schooling as an anti-predator defence in fish. Annales Zoologici Fennici 27:51-66.

Magurran AE. 2005. Evolutionary Ecology: The Trinidadian Guppy: Oxford University Press. Magurran AE, and Girling SL. 1986. Predator model recognition and response habituation in shoaling minnows. Animal Behaviour 34:510-518. http://dx.doi.org/10.1016/S0003-3472(86)80119-1

Magurran AE, and Pitcher TJ. 1987. Provenance, Shoal Size and the Sociobiology of Predator-Evasion Behaviour in Minnow Shoals. Proceedings of the Royal Society of London Series B Biological Sciences 229:439-465. 10.1098/rspb.1987.0004

Magurran AE, and Seghers BH. 1994. Predator Inspection Behaviour Covaries with Schooling Tendency Amongst Wild Guppy, Poecilia reticulata, Populations in Trinidad. Behaviour 128:121-134.

Magurran AE, Seghers BH, Shaw PW, and Carvalho GR. 1994. Schooling preferences for familiar fish in the guppy, Poecilia reticulata. Journal of Fish Biology 45:401-406. 10.1111/j.1095-8649.1994.tb01322.x

Marras S, Killen SS, Claireaux G, Domenici P, and McKenzie DJ. 2011. Behavioural and kinematic components of the fast-start escape response in fish: individual variation and temporal repeatability. The Journal of Experimental Biology 214:3102-3110. $10.1242 /$ jeb.056648

Mathis A, and Smith RJF. 1993. Chemical alarm signals increase the survival time of fathead minnows (Pimephales promelas) during encounters with northern pike (Esox Lucius). Behavioral Ecology 4:260-265. 10.1093/beheco/4.3.260

Ojanguren AF, and Braña F. 2003. Effects of size and morphology on swimming performance in juvenile brown trout (Salmo trutta L.). Ecology of Freshwater Fish 12:241-246. 10.1046/j.1600-0633.2003.00016.x

Ojanguren AF, and Fuiman LA. 2010. Seasonal variability in antipredator performance of red drum larvae. Marine Ecology Progress Series 413:117-123. 10.3354/meps08697

Pitcher T. 1991. Who Dares, Wins: the Function and Evolution of Predator Inspection Behaviour in Shoaling Fish. Netherlands Journal of Zoology 42:371-391. doi:https://doi.org/10.1163/156854291X00397 
483

484

485

486

487

488

489

490

491

492

493

494

495

496

497

498

499

500

501

502

503

504

505

506

507

508

509

510

511

512

513

514

515

516

517

518

519

520

521

522

523

524

525

526

527
Reby D, Hewison M, Izquierdo M, and Dominique P. 2001. Red deer (Cervus elaphus) hinds discriminate between the roars of their current harem-holder stag and those of neighbouring stags. Ethology 107:951-959. 10.1046/j.1439-0310.2001.00732.x

Ruxton GD, and Johnsen S. 2016. The effect of aggregation on visibility in open water. Proceedings of the Royal Society B: Biological Sciences 283. 10.1098/rspb.2016.1463

Strodl MA, and Schausberger P. 2012. Social Familiarity Reduces Reaction Times and Enhances Survival of Group-Living Predatory Mites under the Risk of Predation. PLoS One 7:e43590. 10.1371/journal.pone.0043590

Strodl MA, and Schausberger P. 2013. Social familiarity relaxes the constraints of limited attention and enhances reproduction of group-living predatory mites. Oikos 122:1217-1226. 10.1111/j.1600-0706.2012.20833.x

Swaney W, Kendal J, Capon H, Brown C, and Laland KN. 2001. Familiarity facilitates social learning of foraging behaviour in the guppy. Animal Behaviour 62:591-598. 10.1006/anbe.2001.1788

Tanner CJ, and Keller L. 2012. Nest distribution varies with dispersal method and familiarity-mediated aggression for two sympatric ants. Animal Behaviour 84:11511158. http://dx.doi.org/10.1016/j.anbehav.2012.08.018

Team RDC. 2016. R: A language and environment for statistical computing. R. R Foundation for Statistical Computing. R 2.15.3 ed. Vienna, Austria: R Foundation for Statistical Computing.

Thibault RE. 1974. Genetics of cannibalism in a viviparous fish and its relationship to population density. Nature Nature 251:138 - 140.

Vilhunen S, and Hirvonen H. 2003. Innate antipredator responses of Arctic charr (Salvelinus alpinus) depend on predator species and their diet. Behavioral Ecology and Sociobiology 55:1-10. 10.1007/s00265-003-0670-8

Ward AJW, and Hart PJB. 2003. The effects of kin and familiarity on interactions between fish. Fish and Fisheries 4:348-358. 10.1046/j.1467-2979.2003.00135.x

Ward AJW, Herbert-Read JE, Sumpter DJT, and Krause J. 2011. Fast and accurate decisions through collective vigilance in fish shoals. Proceedings of the National Academy of Sciences 108:2312-2315. 10.1073/pnas.1007102108

Ward AJW, and Webster M. 2016. Sociality: The Behaviour of Group-Living Animals: Springer International Publishing.

Webb PW. 1978. Fast-start Performance and Body Form in Seven Species of Teleost Fish. The Journal of Experimental Biology 74:211-226.

Weihs D. 1973. The mechanism of rapid starting of slender fish. Biorheology 10:343-350.

Weihs D, and Webb PW. 1984. Optimal avoidance and evasion tactics in predator-prey interactions. Journal of Theoretical Biology 106:189-206. http://dx.doi.org/10.1016/0022-5193(84)90019-5

Wisenden BD, and Smith RJF. 1998. A re-evaluation of the effect of shoalmate familiarity on the proliferation of alarm substance cells in ostariophysan fishes. Journal of Fish Biology 53:841-846. 10.1111/j.1095-8649.1998.tb01837.x

Ydenberg RC, and Dill LM. 1986. The Economics of Fleeing from Predators. Advances in the Study of Behavior 16:229-249. http://dx.doi.org/10.1016/S0065-3454(08)60192-8

Zach GJ, Peneder S, Strodl MA, and Schausberger P. 2012. Social familiarity governs prey patch-exploitation, - leaving and inter-patch distribution of the group-living 
$528 \quad$ predatory mite Phytoseiulus persimilis. PLoS One 7:e42889.

$529 \quad 10.1371 /$ journal.pone.0042889

530 Zajitschek Susanne RK, and Brooks Robert C. 2008. Distinguishing the Effects of Familiarity, 531 Relatedness, and Color Pattern Rarity on Attractiveness and Measuring Their Effects 532 on Sexual Selection in Guppies (Poecilia reticulata). The American Naturalist

$533 \quad 172: 843-854.10 .1086 / 593001$

534 


\section{Figure 1}

Figure 1

Diagram of the two experimental treatments (familiar and unfamiliar). Individuals were allocated to a holding tank with two other conspecifics for two weeks. Each testing day, three groups were tested where fish remained with those they had been sharing a tank with (familiar treatment). The other three groups had the individuals swapped so that none of the fish had encountered each other previously (unfamiliar treatment). Forty-two groups were tested in total, 21 of each treatment.

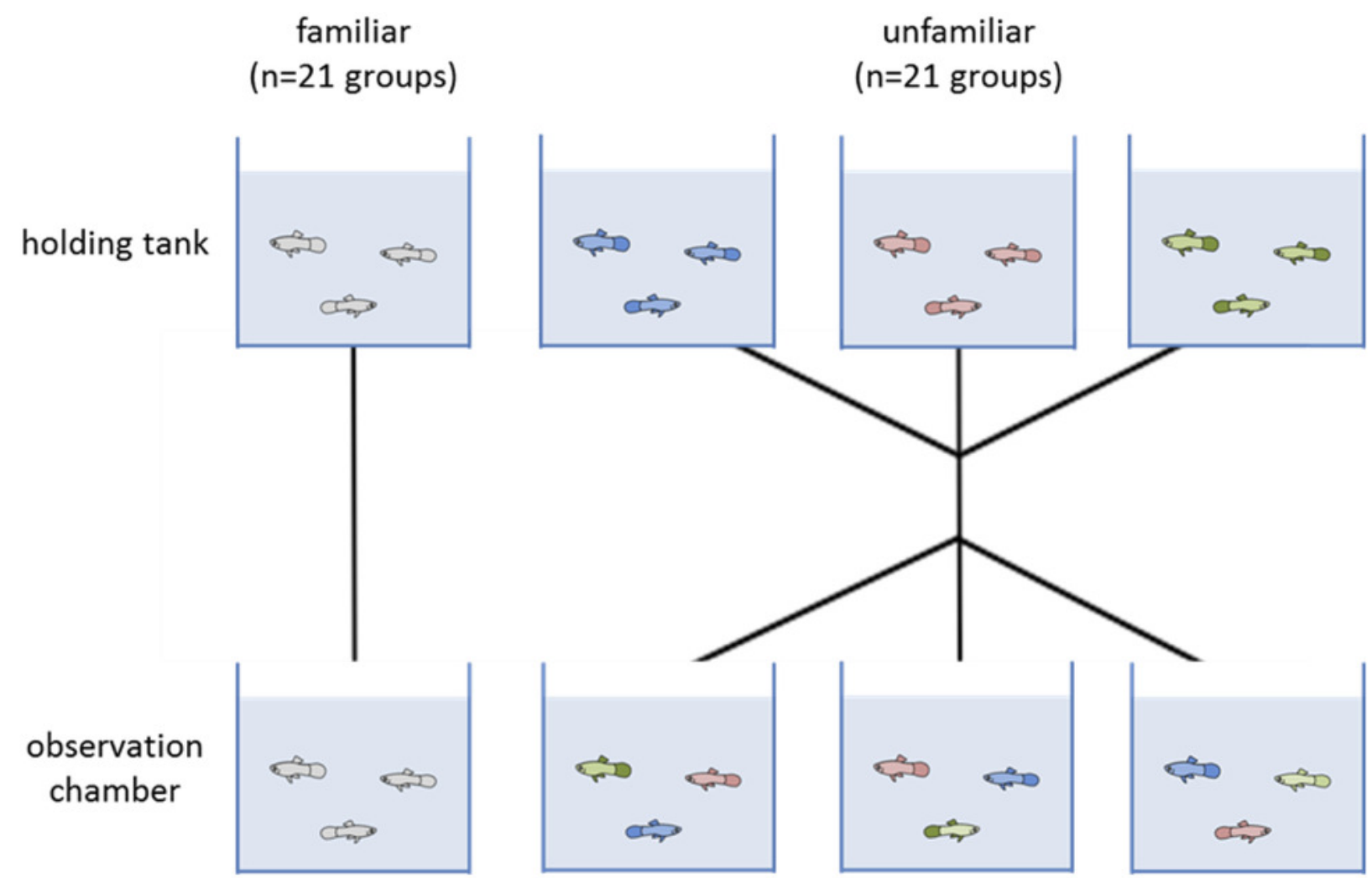




\section{Figure 2}

Figure 2

Illustration of the experimental setup. A camera was placed $1 \mathrm{~m}$ away from a glass tank ( $10 \mathrm{x}$ $10 \times 10 \mathrm{~cm}$ ) positioned before the LDC screen that showed the digital display of a looming object. The front of the tank and the overhead view of the tank were recorded in high-speed video for each trial. The distance in centimetres of the digital looming object was displayed on the top left of the screen.

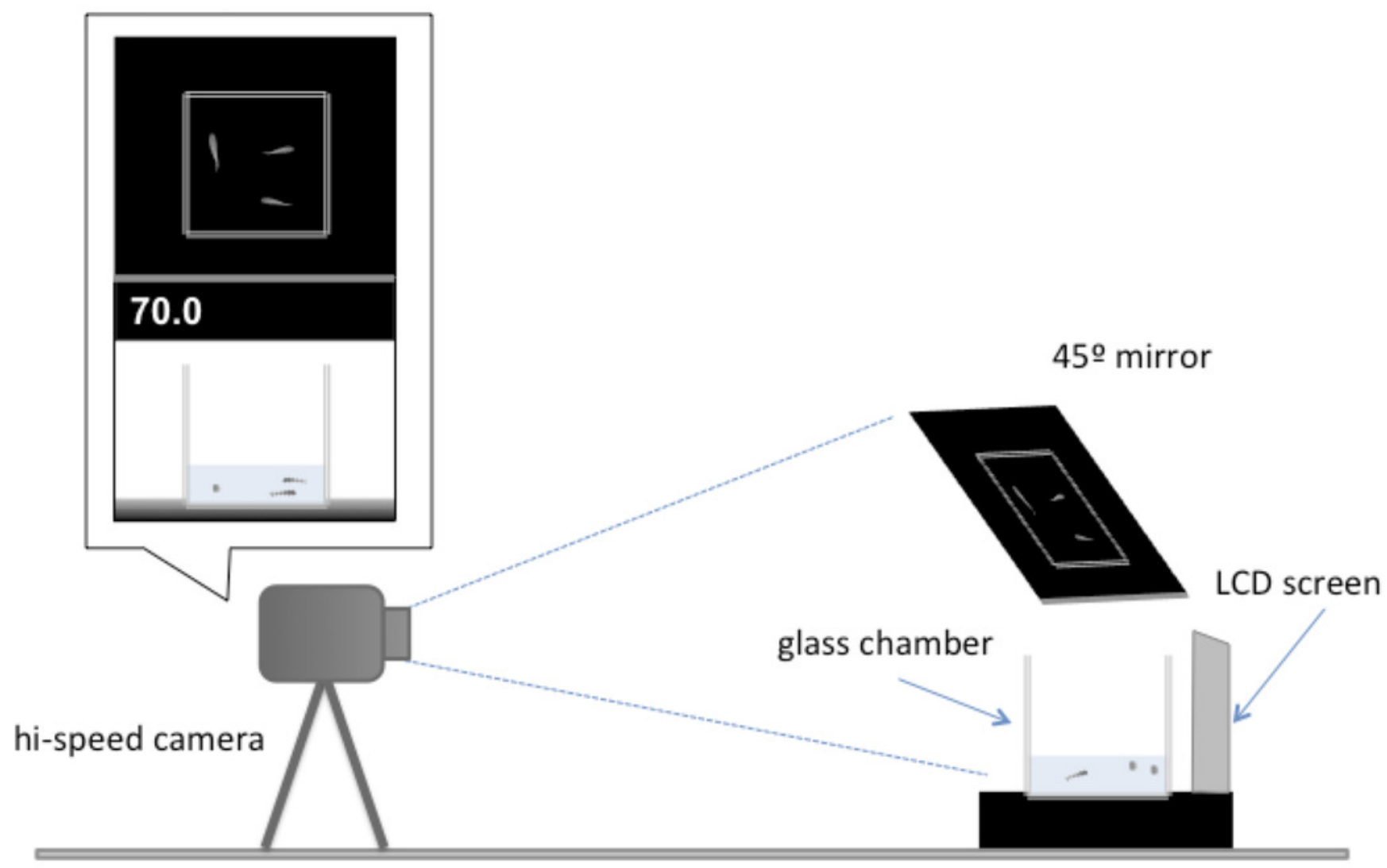


Figure 3

Figure 3

Responsiveness for familiar and unfamiliar groups in terms of how many individuals in a group of three responded to the stimulus. The numbers within the bubbles give the number of groups.

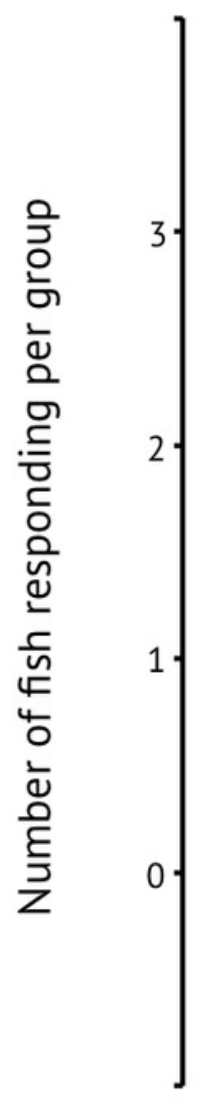

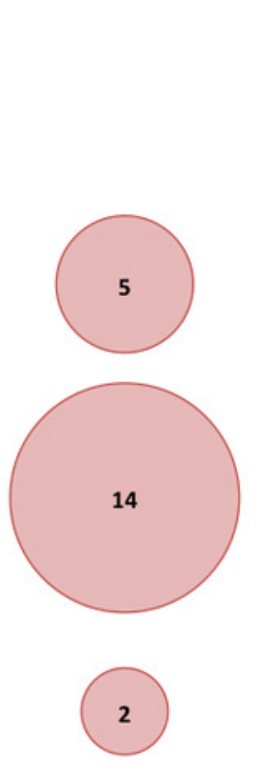
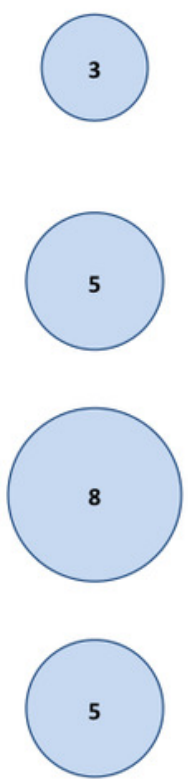

Familiar

Unfamiliar 


\section{Figure 4 (on next page)}

\section{Figure 4}

Variation in reactive distance (A), maximum speed (B), maximum acceleration (C) and total distance (D), in familiar (open circles) and unfamiliar (closed circles) groups. Lines were fitted using the coefficients of linear models. 
PeerJ
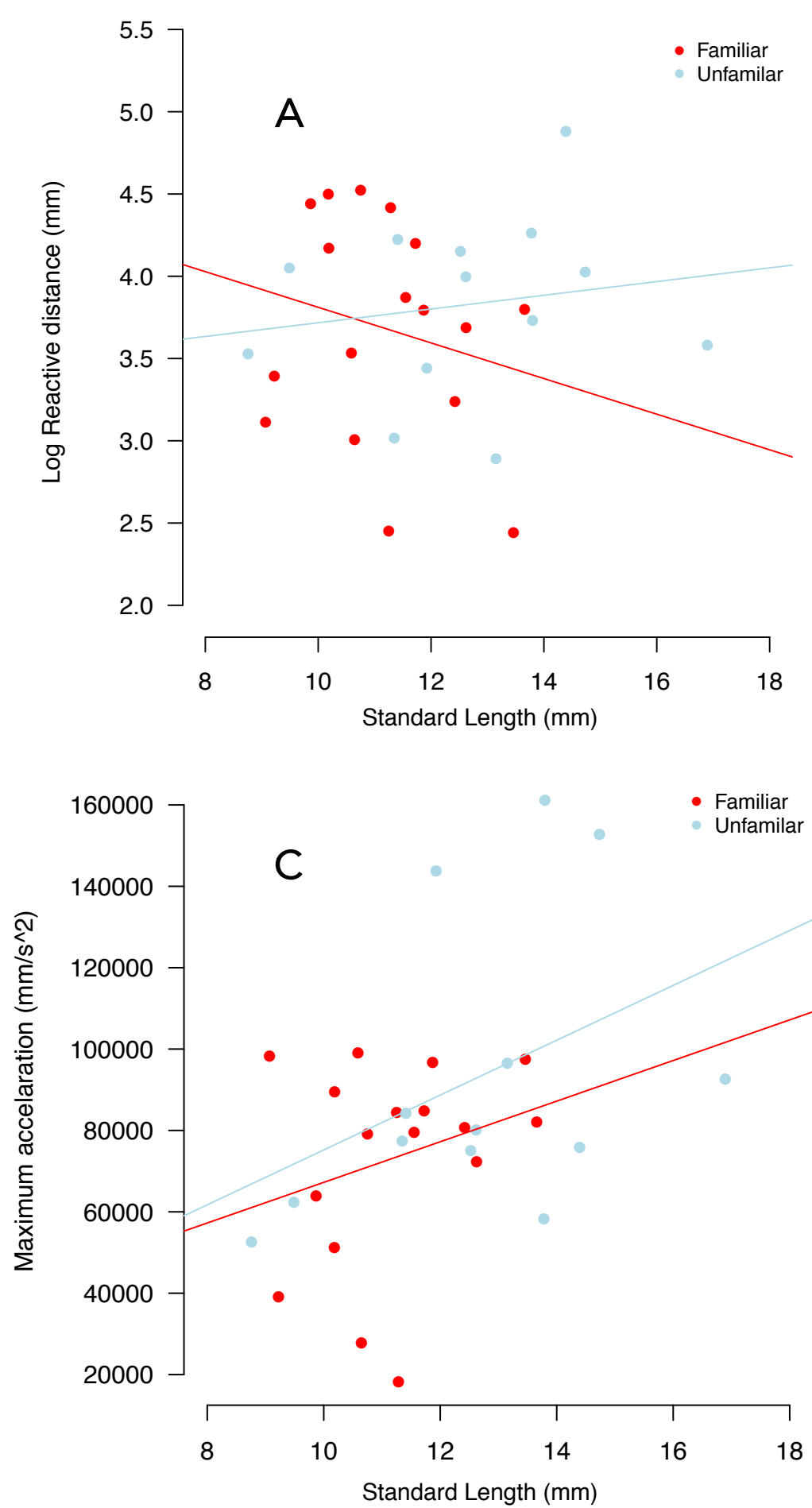

Peer) reviewing PDF | (2017:04:17311:3:0:NEW 14 Sep 2017)
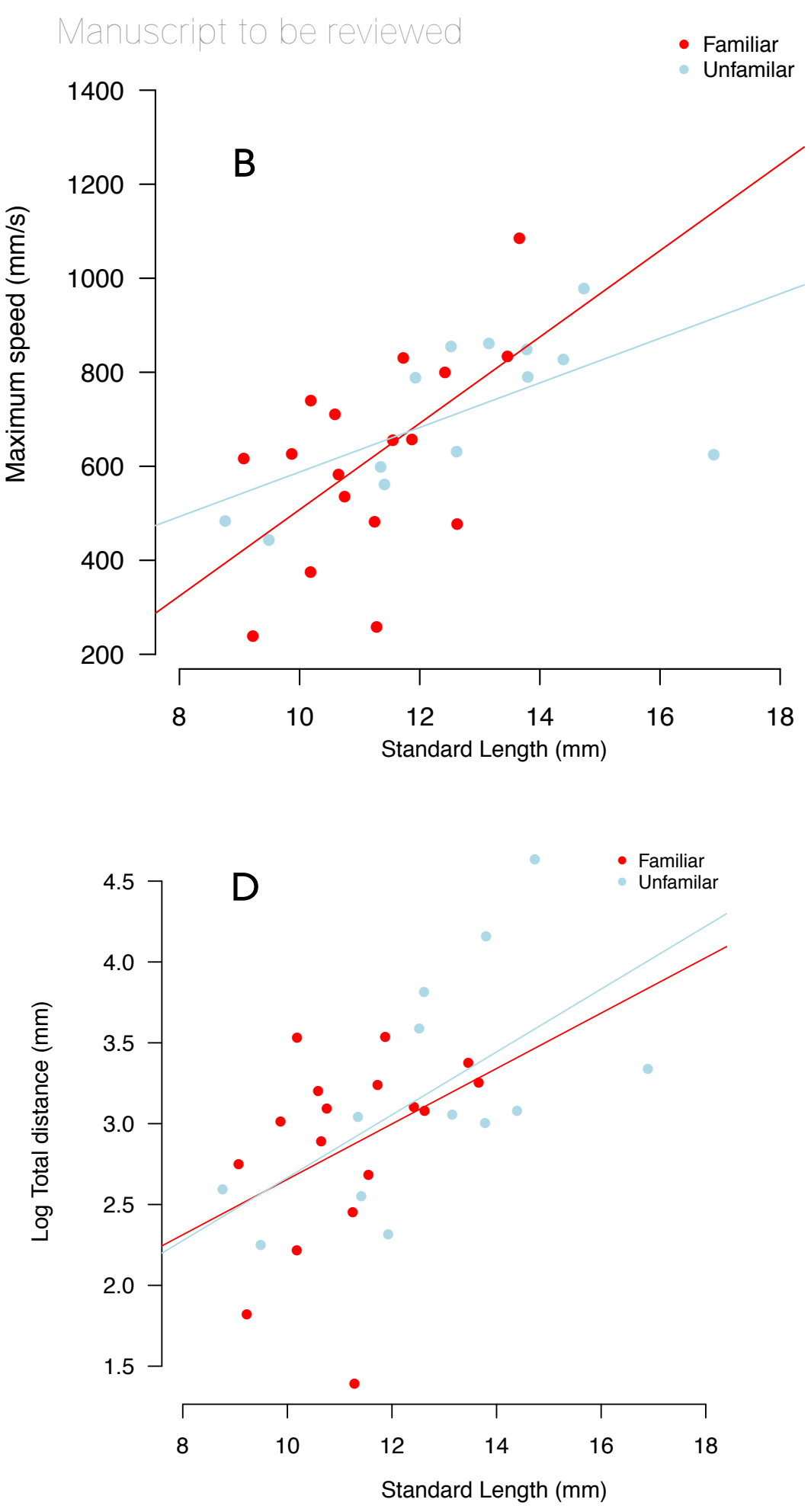


\section{Table 1 (on next page)}

Table 1

Generalised linear models for testing the effect of familiarity on different qualitative measures of response. 
1

2

3Table 1

4

\begin{tabular}{llcccc}
\hline Response variable & \multicolumn{1}{c}{$\begin{array}{c}\text { Explanatory } \\
\text { variable }\end{array}$} & Estimate & $\begin{array}{c}\text { Std Error } \\
\mathrm{Sq}\end{array}$ & T-value & p-value \\
\hline & & & & & \\
& Intercept & 2.526 & 1.819 & 1.389 & 0.175 \\
\multirow{2}{*}{ Reactive distance } & Unfamiliar & 1.985 & 2.879 & 0.690 & 0.496 \\
& Length & 0.069 & 0.142 & 0.488 & 0.629 \\
& Unfamiliar: length & -0.178 & 0.243 & -0.737 & 0.467 \\
\hline
\end{tabular}

\begin{tabular}{llcccc} 
& Intercept & 175.7 & 269.9 & 0.651 & 0.519 \\
\multirow{2}{*}{ Maximum speed } & Familiar & -487.8 & 427.2 & -1.140 & 0.263 \\
& Length & 44.14 & 21.16 & 2.086 & 0.045 \\
& Familiar: length & 37.91 & 36.06 & 1.051 & 0.301 \\
\hline
\end{tabular}

\begin{tabular}{clcccc} 
Maximum & Intercept & -147.6 & 4846 & -0.003 & 0.997 \\
acceleration & Familiar & 2989 & 7671 & 0.390 & 0.699 \\
& Length & 6909 & 3799 & 1.819 & 0.078 \\
& Familiar: length & -3032 & 6473 & -0.468 & 0.642 \\
\hline
\end{tabular}

\begin{tabular}{llllll} 
& Intercept & 0.699 & 0.908 & 0.771 & 0.447 \\
Total distance & Familiar & 0.562 & 1.437 & 0.391 & 0.698 \\
& Length & 0.194 & 0.071 & 2.733 & 0.010 \\
& Familiar: length & -0.055 & 0.121 & -0.454 & 0.653 \\
\hline 5 & & & & & \\
7 & & & & &
\end{tabular}

